DRAFT VERSION OCTOBER 28, 2018

Preprint typeset using LTEX style emulateapj v. 08/22/09

\title{
STRONG MASS SEGREGATION AROUND A MASSIVE BLACK HOLE
}

\author{
TAl AleXAnder* \\ Faculty of Physics, Weizmann Institute of Science, P.O. Box 26, Rehovot 76100, Israel \\ AND \\ Clovis HopMAN \\ Leiden University, Leiden Observatory, P.O. box 9513, NL-2300 RA Leiden, The Netherlands \\ Draft version October 28, 2018
}

\begin{abstract}
We show that the mass-segregation solution for the steady state distribution of stars around a massive black hole $(\mathrm{MBH})$ has two branches: the known weak segregation solution (Bahcall \& Wolf 1977), and a newly discovered strong segregation solution, presented here. The nature of the solution depends on the heavy-tolight stellar mass ratio $M_{H} / M_{L}$ and on the unbound population number ratio $N_{H} / N_{L}$, through the relaxational coupling parameter $\Delta=4 N_{H} M_{H}^{2} /\left[N_{L} M_{L}^{2}\left(3+M_{H} / M_{L}\right)\right]$. When the heavy stars are relatively common $(\Delta \gg 1)$, they scatter frequently on each other. This efficient self-coupling leads to weak mass segregation, where the stars form $n \propto r^{-\alpha_{M}}$ mass-dependent cusps near the MBH, with indices $\alpha_{H}=7 / 4$ for the heavy stars and $3 / 2<\alpha_{L}<7 / 4$ for the light stars (i.e. $\max \left(\alpha_{H}-\alpha_{L}\right) \simeq 1 / 4$ ). However, when the heavy stars are relatively rare $(\Delta \ll 1)$, they scatter mostly on light stars, sink to the center by dynamical friction and settle into a much steeper cusp with $2 \lesssim \alpha_{H}<11 / 4$, while the light stars form a $3 / 2<\alpha_{L}<7 / 4$ cusp, resulting in strong segregation (i.e. $\max \left(\alpha_{H}-\alpha_{L}\right) \simeq 1$ ). We show that the present-day mass function of evolved stellar populations (coeval or continuously star forming) with a universal initial mass function, separate into two distinct mass scales, $\sim 1 M_{\odot}$ of main sequence and compact dwarfs, and $\sim 10 M_{\odot}$ of stellar black holes (SBHs), and have $\Delta<0.1$. We conclude that it is likely that many relaxed galactic nuclei are strongly segregated. We review indications of strong segregation in observations of the Galactic Center and in results of numeric simulations, and briefly list some possible implications of a very high central concentration of SBHs around a MBH.
\end{abstract}

Subject headings: Galaxy: kinematics and dynamics — stellar dynamics — black hole physics

\section{INTRODUCTION}

Early theoretical studies of the dynamics and distribution of stars around a MBH (Peebles 1972; Bahcall \& Wolf 1976, 1977; Young 1980) were triggered by the discovery of quasars (e.g. Matthews \& Sandage 1963; Schmidt 1963) and the realization that many galactic nuclei may contain a central massive collapsed object (Lynden-Bell 1969; Wolfe \& Burbidge 1970), as well as by the discovery of X-ray sources in globular clusters (Giacconi et al. 1972), which were then thought to be accreting MBHs (e.g. Wyller 1970; Bahcall \& Wolf 1976; see also Miller \& Hamilton 2002 for a recent reevaluation of this possibility). The main motivations for these studies were the prospect of detecting MBHs by the observed stellar density profile and by tidal disruption flares, and the possible role of tidal disruptions of stars in the growth of MBHs (Lightman \& Shapiro 1977; Cohn \& Kulsrud 1978; Shapiro \& Marchant 1978; Rees 1988)

The renewed interest in this problem is driven by observations of stars closely orbiting the Galactic $\mathrm{MBH}$ (Eisenhauer et al. 2005; Ghez et al. 2005) and the accumulating data on their distribution and dynamics (Alexander 1999; Genzel et al. 2000, 2003; Schödel et al. 2003, 2007), as well as by the prospects of detecting gravitational waves (GW) from extreme mass ratio inspiral sources by future GW detectors (EMRIs: compact remnants inspiraling into MBHs, see review by Amaro-Seoane et al. 2007; Hopman 2006). EMRI rates and properties strongly depend on the stellar density and the stellar dynamical processes within

*Incumbent of the William Z. \& Eda Bess Novick career development chair Electronic address: tal.alexander@weizmann.ac.il

Electronic address: clovis@strw.leidenuniv.nl
$O(0.01 \mathrm{pc})$ of the $\mathrm{MBH}$, where inspiraling sources originate (e.g. Hopman \& Alexander 2005, 2006a).

Mass segregation occurs in dynamically relaxed systems. MBHs are naturally expected to lie in relaxed cores in scenarios where the $\mathrm{MBH}$ is formed by run-away mergers in the extreme central density following core collapse (Rees 1984), which occurs on timescales much longer then the relaxation time, $T_{R}$ (e.g. Spitzer 1987; Quinlan 1996; Freitag et al. $2006 \mathrm{~b}$ c). Likewise, the extreme mass ratio targets of the planned Laser Interferometer Space Antenna ${ }^{1} \mathrm{GW}$ detector (LISA) are expected to originate in relaxed nuclei, since LISA design is sensitive to GW from MBHs with mass $\lesssim 10^{7} M_{\odot}$. The observed correlation between the MBH mass $\widetilde{M}_{\bullet}$ and the typical velocity dispersion of the spheroid of the host galaxy, $M_{\bullet} \propto \sigma^{\beta}, 4 \lesssim \beta \lesssim 5$ (the $M_{\bullet} / \sigma$ relation, Ferrarese \& Merritt 2000; Gebhardt et al. 2000) then implies that such nuclei are dynamically relaxed and very dense (Alexander 2007; Merritt et al. 2007). This can be seen by assuming for simplicity $\beta=4$ (a higher value only reinforce these conclusions), and noting that the MBH radius of influence $r_{h} \sim G M_{\bullet} / \sigma^{2} \propto$ $M_{\bullet}^{1 / 2}$ encompasses a stellar mass of order $M_{\bullet}$, so that the number of stars there is $N_{h} \sim M_{\bullet} / M_{\star}$, where $M_{\star}$ is the typical stellar mass, and the mean stellar density is $\bar{n}_{h} \sim N_{h} / r_{h}^{3} \propto$ $M_{\bullet}^{-1 / 2}$. The " $n v \Sigma$ " rate estimate of strong gravitational collisions then implies that $T_{R}^{-1}\left(r_{h}\right) \sim \bar{n}_{h} \sigma\left(G M_{\star} / \sigma^{2}\right)^{2} \propto M_{\bullet}^{-5 / 4}$. A more rigorous estimate shows that for the Galactic $\mathrm{MBH}$ $\left(M_{\bullet} \simeq 4 \times 10^{6} M_{\odot}\right.$, Eisenhauer et al. 2005; Ghez et al. 2005),

\footnotetext{
${ }^{1}$ See LISA mission website http://lisa.nasa.gov
} 
an archetype of LISA targets, $T_{R} \sim O(1 \mathrm{Gyr})<t_{H}$ (the Hubble time) and $\bar{n}_{h} \sim O\left(10^{5} \mathrm{pc}^{-3}\right)$. The density in the stellar cusp near the $\mathrm{MBH}$ is orders of magnitude higher still (see below). Since $T_{R} \propto M_{\bullet}^{-5 / 4}$, isolated nuclei with $M_{\bullet} \lesssim 10^{7} M_{\odot}$ are predicted to be relaxed.

A single mass stellar system around a $\mathrm{MBH}$ is expected to relax to a $r^{-\alpha}$ cusp with $\alpha=7 / 4$ (Bahcall \& Wolf 1976). This results from the fact that the gravitational orbital energy gained by the system when stars are destroyed near the MBH is conserved as it is shared and carried outward by the remaining stars, $\dot{E}(r) \sim E(r) N(<r) / T_{R} \propto r^{-1} r^{3-\alpha} / r^{\alpha-3 / 2}=$ $r^{7 / 2-2 \alpha}=$ const (Binney \& Tremaine 1987). When the system includes a spectrum of masses, $M_{L} \leq M \leq M_{H}$, the approach toward equipartition by 2-body interactions decreases the specific kinetic energy of the high-mass stars, while that of the low-mass stars increases. As a result, the high-mass stars sink and concentrate in the center on the dynamical friction timescale $T_{\mathrm{df}} \sim T_{R}\langle M\rangle / M_{H}$, while the low-mass stars float out (Spitzer 1987).

Bahcall \& Wolf (1977, hereafter BW77) approximated the mass segregation problem in the Fokker-Planck formalism, and solved for the steady state, angular momentum averaged stellar distribution functions (DFs) $f_{M}(E)$. They found that near the $\mathrm{MBH}$, the DFs can be approximated by powerlaws, $f_{M}(E) \propto E^{p_{M}}$, and that the stellar current into the $\mathrm{MBH}$, $Q_{M}(E)$, is very small, which leads to a specific relation between the stellar mass and the logarithmic slope of the DF, $p_{M}=M / 4 M_{H}$. In the Keplerian limit near the $\mathrm{MBH}$, these DFs correspond to power-law density cusps, with $\alpha_{M}=3 / 2+$ $p_{M}$. The BW77 solution thus predicts a relatively small range of central concentrations, from $\alpha_{H}=7 / 4$ for the heaviest stars in the populations, to $\alpha_{L} \rightarrow 3 / 2$ for the lightest stars. We show below that the "zero-flow" assumption breaks down when the massive stars are too rare to scatter efficiently against each other, and instead sink to the center by dynamical friction against the light stars. As a result, the BW77 relation between $M$ and $p_{M}$ no longer holds, and the range of central concentrations far exceeds that of the BW77 solution.

The Galactic Center (GC) provides to date the few available observations that directly bear on the question of mass segregation around a $\mathrm{MBH}$. The over-abundance of X-ray transients in the central $\sim 1$ pc of the GC was interpreted as evidence of a high central concentration of neutron stars and stellar black holes (SBHs) (Muno et al. 2005); The central decrease in the surface density of the low-mass horizontal branch "red clump" giants was interpreted as evidence of the evacuation of long-lived light objects by mass segregation (Levi 2006; Schödel et al. 2007). The dynamical upper limit on the distributed dark mass in the inner few mpc around the galactic $\mathrm{MBH}, M_{\mathrm{dm}} / M_{\bullet} \sim$ few $\times 0.01$ (Mouawad et al. 2005; Gillessen et al. 2008; Ghez et al. 2008), is still 10-100 times larger than predicted by approximate theoretical estimates (Morris 1993; Miralda-Escudé \& Gould 2000), FokkerPlanck calculations (Hopman \& Alexander 2006b), or by the conservative drain limit (Alexander \& Livio 2004), which places an upper bound on the maximal number of compact remnants that can avoid being thrown into the $\mathrm{MBH}$ by mutual 2-body scattering. These theoretical estimates are consistent with the upper bound derived from the observed limits on the diffuse X-ray in the central 0.7 pc $\left(\sim 2 \times 10^{4}\right)$ (Deegan \& Nayakshin 2007).

Detailed analysis of observations of the GC (Levi 2006; Schödel et al. 2007) suggests that the degree of segregation between the light and heavy stars is stronger than expected in the BW77 solution, although this interpretation of the data is not unique. Strong segregation in the GC is further supported by analytic (Hopman \& Alexander 2006b) and Monte-Carlo (Freitag et al. 2006a, M. Freitag, priv. comm.) mass segregation results (Fig. 1). These calculations do not take into account star formation and evolution, but instead assume for simplicity a non-evolving mass function that is based on a model of the GC's present day mass mass function (PMF) as an old, continuously star forming population with the "universal" initial mass function (IMF) (Alexander \& Sternberg 1999; Alexander 2005, table 2.1). In these theoretical models $\alpha_{L} \simeq 1.5$, while $\alpha_{H} \gtrsim 2$ at $r \sim 0.1$ pc. $N$-body simulations of stellar clusters with evolving stellar populations and a central intermediate mass black hole (IMBH) also provide hints of strong mass segregation (Baumgardt et al. 2004, J. Makino, priv. comm.). As we show below (Fig. 4), these results reflect the fact that the relative fraction of SBHs in old populations is below a critical threshold needed for them to scatter efficiently against each other and maintain the weak segregation solution. Instead, they sink to the center by dynamical friction and settle into the strong segregation solution.

This paper is organized as follows. The Fokker-Planck formulation of the mass segregation problem, the choices of boundary conditions and the various simplifying approximations are described in $\$ 2$ The physical meaning of the relaxational coupling parameter, $\Delta$, is discussed in $\$ 3$. The $\Delta$ parameters of different stellar populations are explored in \$4 A large grid of Fokker-Planck mass segregation calculations is presented and compared to simple analytic estimates in $\$ 5$ The results and their implications are discussed and summarized in $\$ 6$

\section{THE ENERGY DIFFUSION FOKKER-PLANCK EQUATION}

The MBH dominates the gravitational potential within its radius of influence, where the dynamics are approximately Keplerian. Following the approach introduced by Bahcall \& Wolf (1976, 1977) and its implementation by Hopman \& Alexander (2006b), we calculate the approximate steady state DFs of a non-evolving stellar population in a spherical Keplerian system around a fixed MBH. This is done by solving the time and energy-dependent, angular momentum-averaged particle conservation equation that describes the 2-body diffusion of stars in energy from a fixed unbound reservoir into the MBH sink (resonant relaxation, Rauch \& Tremaine 1996, is not taken into account). One of the stellar mass groups, of mass $M_{\star}$, is taken to be the reference star. It is assumed that unbound stars of mass $M$ with velocity dispersion $\sigma_{M}^{2}$ and specific energy $\varepsilon \equiv \psi-v^{2} / 2<0$ ( $\psi=-\phi$ is the negative of the gravitational potential $\phi$ and $\varepsilon>0$ for bound stars), have a Maxwellian DF,

$$
f_{M}(\varepsilon)=S_{M} \frac{n_{\star}}{\left(2 \pi \sigma_{M}^{2}\right)^{3 / 2}} \exp \left(\varepsilon / \sigma_{M}^{2}\right), \quad(\varepsilon<0),
$$

where $n_{\star}$ is the number density normalization of the reference star. Since the number density of unbound stars

$$
\begin{aligned}
n_{M} & =4 \pi \int_{\sqrt{2 \psi}}^{\infty} v^{2} f_{M}(\varepsilon) \mathrm{d} v=4 \pi \int_{-\infty}^{0} \sqrt{2(\psi-\varepsilon)} f_{M}(\varepsilon) \mathrm{d} \varepsilon \\
& =S_{M} n_{\star}\left[\frac{2}{\sqrt{\pi}} \frac{\sqrt{\psi}}{\sigma_{M}}+\exp \left(\frac{\psi}{\sigma_{M}^{2}}\right) \operatorname{erfc}\left(\frac{\sqrt{\psi}}{\sigma_{M}}\right)\right] \\
& \rightarrow S_{M} n_{\star} \quad\left(\psi / \sigma_{M}^{2} \rightarrow 0\right)
\end{aligned}
$$


the quantity $S_{M} n_{\star}$ can be interpreted as the total density of stars of mass $M$ far from the $\mathrm{MBH}$, where $\psi / \sigma_{M}^{2} \ll 1$ and almost all stars are unbound to the $\mathrm{MBH}$. In that case $S_{M}$ is the asymptotic number density ratio of star $M$ relative to the reference $\operatorname{star}\left(S_{\star}=1\right.$ by definition). The asymptotic population ratios between stars of mass $M_{H}$ and $M_{L}$ are designated below by $N_{H} / N_{L}=S_{H} / S_{L}$. It is assumed that the asymptotic PMF, and its corresponding population ratios $N_{H} / N_{L}$, depend only on the IMF, on the star formation history and on stellar evolution physics, and are independent of mass segregation dynamics, which are effective only near the MBH.

Stellar dynamics near the $\mathrm{MBH}$ are described here in units where $G=1$, mass is measured in units of the mass of the reference star, $M_{\star}$, specific energy in units of its velocity dispersion $\varepsilon_{\star}=\sigma_{\star}^{2}$, and time in units of its two-body relaxation time at the radius of influence,

$$
t_{\star}=\frac{3\left(2 \pi \sigma_{\star}^{2}\right)^{3 / 2}}{32 \pi^{2} G^{2} M_{\star}^{2} n_{\star} \ln \Lambda},
$$

where the Coulomb term is estimated as $\Lambda=M_{\bullet} / M_{\star}$. Phase space density is expressed in units of $f_{\star}=n_{\star} /\left(2 \pi \sigma_{\star}^{2}\right)^{3 / 2}$ and distance in expressed units of the $\mathrm{MBH}$ radius of influence $r_{\star}=G M_{\bullet} / \sigma_{\star}^{2}$. In these units, the dimensionless specific orbital energy is defined as $x=\varepsilon / \sigma_{\star}^{2}=r_{\star} / 2 a$ ( $a$ is the semimajor axis), the dimensionless time is defined as $\tau=t / t_{\star}$, and the dimensionless DF as $g_{M}=f_{M} / f_{\star}$.

The evolution of the dimensionless DF of each stellar mass group, $g_{M}$, is described by the energy flow integral $Q_{M}$, which expresses the change in energy due to 2-body scattering, and the angular momentum-averaged effective loss-cone term $R_{M}$ (Bahcall \& Wolf 1976, 1977),

$$
\frac{\partial}{\partial \tau} g_{M}(x, \tau)=-x^{5 / 2} \frac{\partial}{\partial x} Q_{M}(x, \tau)-R_{M}(x, \tau) .
$$

The flow integral is given by

$$
\begin{aligned}
Q_{M}(x)= & \sum_{M^{\prime}} M M^{\prime} \int_{-\infty}^{x_{D}} \frac{\mathrm{d} x^{\prime}}{\max \left(x, x^{\prime}\right)^{3 / 2}} \times \\
& {\left[g_{M}(x) \frac{\partial g_{M^{\prime}}\left(x^{\prime}\right)}{\partial x^{\prime}}-\frac{M^{\prime}}{M} g_{M^{\prime}}\left(x^{\prime}\right) \frac{\partial g_{M}(x)}{\partial x}\right] . }
\end{aligned}
$$

The averaged effective loss-cone term in the diffusive regime (defined here as $x>x_{\text {diff }}=10$ ) is approximated by (Hopman \& Alexander 2006b)

$$
R_{M}(x)=\frac{g_{M}(x)}{\tau_{r}(x) \ln \left[J_{c}(x) / J_{l c}\right]},
$$

where $J_{c}(x)$ is the maximal (circular) angular momentum for energy $x, J_{l c}$ is the angular momentum of the loss-cone (taken here to be $J_{L S O}=4 G M_{\bullet} / c$, the angular momentum of the last stable orbit for $|\varepsilon| \ll c^{2}$ ), and $\tau_{r} \sim\left[\sum_{M} g_{M}(x) M^{2}\right]^{-1}$ is the approximate 2-body relaxation time. The contribution of the loss-cone in the full loss-cone regime, which typically corresponds to $x \lesssim 1 / 2$, is small (e.g. Sver \& Ulmer 1999), and is therefore neglected here. Bahcall \& Wolf (1976, 1977) argued that the loss-cone term has only a small effect on the energy dependence of the DF, and neglected it in their multimass models. In order to allow direct comparison of our calculations with their results, we omit here the loss-cone term as well, unless stated otherwise.
Equation (4) is integrated in time from an arbitrary initial DF until steady-state is achieved, subject to the boundary conditions that the DF falls to zero at some very high energy $x_{D}$ where the stars are destroyed, and that the unbound stars are replenished from a Maxwellian reservoir,

$$
g_{M}\left(x>x_{D}\right)=0, \quad g_{M}(x<0)=C_{M} \exp \left[\left(\sigma_{\star}^{2} / \sigma_{M}^{2}\right) x\right],
$$

where the constant $C_{M}$ is related to the population ratio $S_{M}$ by (see Eq. 1)

$$
C_{M}=\left(\sigma_{\star} / \sigma_{M}\right)^{3} S_{M} .
$$

Specifically, if the different unbound mass groups are in equipartition (as assumed by BW77), then $C_{M}=$ $\left(M / M_{\star}\right)^{3 / 2} S_{M}$. Conversely, if the unbound stellar population has achieved equilibrium by violent relaxation (Lynden-Bell 1967), then all mass groups are expected to have similar velocity dispersions, and $C_{M}=S_{M}=S_{\star}$. Here we assume violent relaxation boundary conditions ${ }^{2}$, since these are suggested by the lack of an observed correlation between scale height and spectral type of old stars in the Milky Way (Kroupa et al. 1993), by the absence of an observed vertical color gradient in edge-on disk galaxies (de Grijs \& Peletier 2000), and by the weak observed spatial color gradients in elliptical galaxies (e.g. Boroson et al. 1983; Cohen 1986). We confirm the conclusion of BW77 that the steady state DFs do not depend strongly on these specific choices of boundary conditions (cf comparison with BW77 equipartition models in Fig. (4).

The spatial density profile that corresponds to the DF is

$$
n_{M}(r)=\frac{2}{\sqrt{\pi}} n_{\star} \int_{-\infty}^{r_{\star} / r} g_{M}(x) \sqrt{r_{\star} / r-x} \mathrm{~d} x .
$$

\section{THE RELAXATIONAL COUPLING PARAMETER}

As shown below in $\$ 5$, the nature of the mass segregation solution, weak or strong, is determined by one parameter, which expresses the relative strength of $M_{H}-M_{H}$ and $M_{H}-M_{L}$ interactions. These can be quantified by the corresponding diffusion coefficients, $D\left(\Delta v_{\|}\right), D\left(\Delta v_{\|}^{2}\right)$ and $D\left(\Delta v_{\perp}^{2}\right)$, which enter the Fokker-Planck equation (e.g. Binney \& Tremaine 1987, Eq. 8-68). The two quadratic scattering coefficients are similar up to an order unity factor, and are approximated here as equal, $D\left(\Delta v_{\|}^{2}\right) \sim D\left(\Delta v_{\perp}^{2}\right) \equiv D\left(\Delta v^{2}\right) / 2$. In a 2-mass system we represent these coefficients for brevity by the notation $D_{S T p}$ where $S=L, H$ is the scattering star (light or heavy), $T=L, H$ is the target star, and $p=s, f$ is the process (scattering, $D\left(\Delta v^{2}\right)$, or dynamical friction, $D\left(\Delta v_{\|}\right)$). There are 8 possible permutations of the diffusion coefficients, $(\{L, H\} \times\{L, H\} \times\{s, f\})$, however, since $D_{S T s} \propto 2 N_{S} M_{S}^{2}$ and $D_{S T f} \propto N_{S} M_{S}\left(M_{S}+M_{T}\right)$, there are only 4 distinct combinations of the mass and number ratios ${ }^{3}$.

2 Eqs. (7) 8) generalize the definition of $g_{M}(x)$ and correct an error in the relation between $C_{M}$ and the population ratio, which appeared in Hopman \& Alexander (2006b). The density profile presented in Hopman \& Alexander (2006b) closely approximates the violent relaxation solution (cf Fig. 11 here), and not, as stated there erroneously, the isothermal solution.

3 The quadratic scattering terms do not depend on the mass of the scattered target star because acceleration by a given gravitational force is equal for all masses. The quadratic terms express a random walk in velocity due to interactions with field stars on a timescale of $T_{R} \sim v^{2} / D\left(\Delta v^{2}\right)$. In contrast, the first order dynamical friction term expresses the back-reaction of the field stars to the target star, and therefore does depend on its mass. The first-order term expresses a systematic deceleration on a timescale of $T_{\mathrm{df}} \sim v / D\left(\Delta v_{\|}\right)$. 

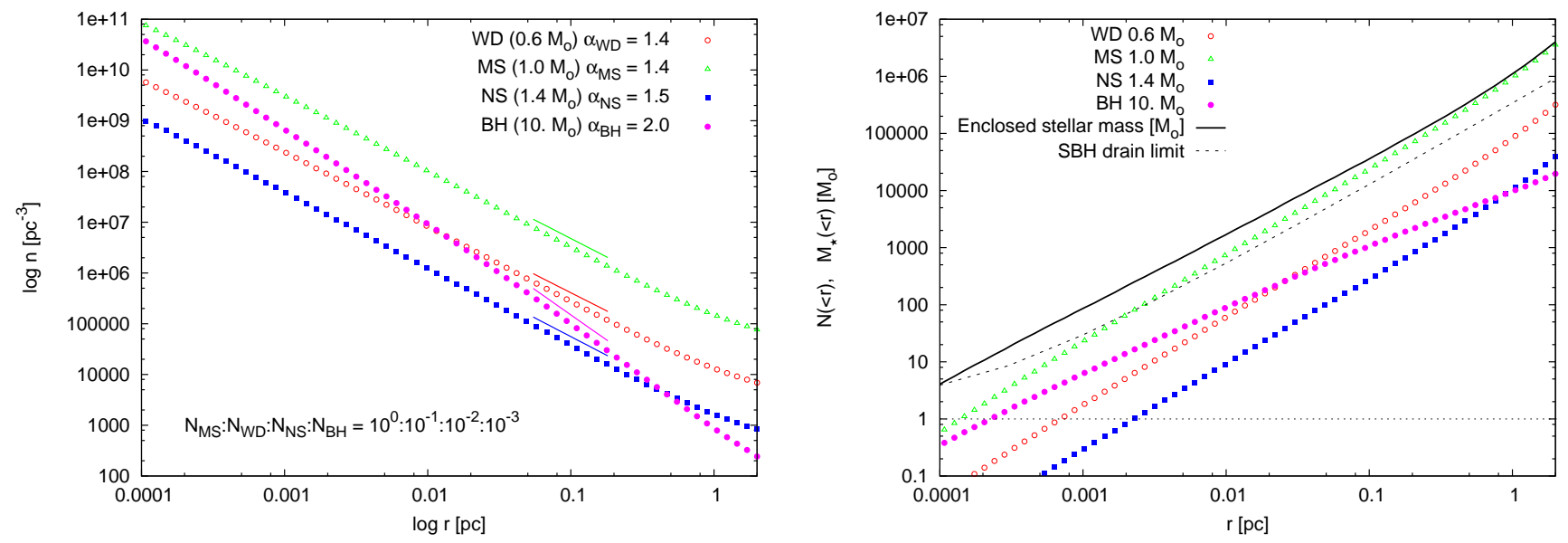

FIG. 1.- A Fokker-Planck model of the GC assuming a non-evolving PMF based on the continuous star-forming model shown in Fig. 22, with violent relaxation boundary conditions, a loss-cone term (Hopman \& Alexander 2006b) and assuming $M_{\bullet}=4 \times 10^{6} M_{\odot}, M_{\star}=1 M_{\odot}, r_{\star}=2 \mathrm{pc}$, and a total stellar mass $M_{\bullet}$ within $r_{\star}$. Left: The spatial number density of the 4 mass components (Eq. 9). Also shown are the best-fit logarithmic slopes around $r=0.1 \mathrm{pc}$. Right: The corresponding enclosed stellar number and total stellar mass as function of radius. The SBH number is consistent with the drain limit (Alexander \& Livio 2004), also shown. The enclosed number of SBHs within $0.1 \mathrm{pc}(0.01 \mathrm{pc})$ is approximately $1.2 \times 10^{3}\left(1.1 \times 10^{2}\right)$, of neutron stars $3.2 \times 10^{2}(12)$, of main sequence stars $2.7 \times 10^{4}\left(9.4 \times 10^{2}\right)$ and of white dwarfs $2.3 \times 10^{3}(76)$.

The relaxational coupling parameter $\Delta$ describes the competition between the self-coupling of the heavy stars and the light-heavy coupling in terms of global properties of the system, the mass and number ratios,

$$
\Delta \equiv \frac{D_{H H s}+D_{H H f}}{D_{L H s}+D_{L H f}} \simeq \frac{N_{H} M_{H}^{2}}{N_{L} M_{L}^{2}} \times \frac{4}{3+M_{H} / M_{L}} .
$$

The definition of $\Delta$ can in principle be generalized to a multi-mass stellar population by specifying the light/heavy mass boundary, $M_{L / H}$ and performing a weighted integration over the mass function ${ }^{4}$. However, as shown in $\$ 4$ below, an evolved stellar population (coeval or continuously starforming) such as is expected near a $\mathrm{MBH}$, is well approximated by a 2-mass system for this purpose.

\subsection{Asymptotic mass segregation limits}

The weak segregation limit (the Bahcall-Wolf solution). In the limit $\Delta \rightarrow \infty$, which is the zero-flow $\left(Q_{M} \rightarrow 0\right)$ limit $(\$ 5)$, the heavy stars dominate the population and relax to the single mass cusp $\alpha_{H}=7 / 4\left(p_{H}=1 / 4\right)$. The light stars heat by scattering against the effectively infinite reservoir of heavy stars and diffuse to higher energies, thereby settling to a flatter cusp with $\alpha_{L} \rightarrow 3 / 2\left(p_{L} \rightarrow 0\right)$.

The strong segregation limit. In the limit $\Delta \rightarrow 0$ and when $M_{H} \gg M_{L}$, the light stars behave as a single mass population with $\alpha_{L}=7 / 4\left(p_{L}=1 / 4\right)$. The rare heavy stars sink to the center by dynamical friction against the effectively infinite reservoir of light stars. Their steady state distribution can be simply derived by noting that the dynamical friction force is approximately $F \propto \rho_{L} / v^{2} \propto r^{1-\alpha_{L}}$, where $v \propto r^{-1 / 2}$ is the Keplerian velocity (Chandrasekhar 1943). The torque on the heavy star (assuming a circular Keplerian orbit, $L \propto r^{1 / 2}$ ) is then $\dot{L} \propto F r \propto r^{2-\alpha_{L}} \propto r^{-1 / 2} \dot{r}$, so that $\dot{r} \propto r^{5 / 2-\alpha_{L}}$. In steady state, the heavy star current, $\dot{N}_{H}$, is independent of radius,

${ }^{4}$ E.g. $\Delta=4\left\langle N M^{2}\right\rangle_{H} /\left(3\left\langle N M^{2}\right\rangle_{L}+\left\langle M_{H}\right\rangle\langle N M\rangle_{L}\right)$, where for a normalized PMF $\mathrm{d} N / \mathrm{d} M,\left\langle N M^{k}\right\rangle_{L, H}=\int_{M_{L}, M_{L / H}}^{M_{L / H}, M_{H}} M^{k}(\mathrm{~d} N / \mathrm{d} M) \mathrm{d} M$, and $\left\langle M_{H}\right\rangle=$ $\int_{M_{L / H}}^{M_{H}} M(\mathrm{~d} N / \mathrm{d} M) \mathrm{d} M / \int_{M_{L / H}}^{M_{H}}(\mathrm{~d} N / \mathrm{d} M) \mathrm{d} M$.

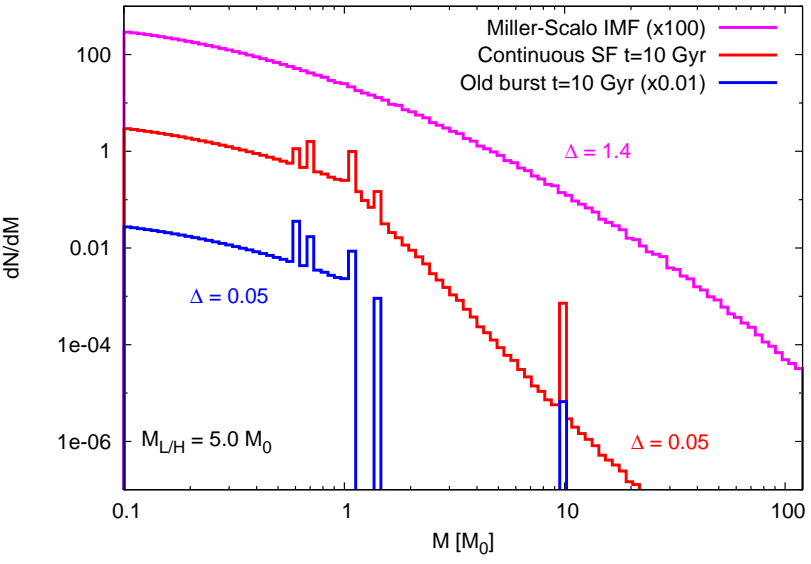

FIG. 2.- The PMF and the relaxation coupling parameter for the Miller-Scalo IMF (Miller \& Scalo 1979) (top line) assuming 10 Gyr of continuous star formation (middle line) or a $10 \mathrm{Gyr}$ old coeval population (bottom line). The IMF was evolved using the Solar metallicity stellar tracks of Schaller et al. (1992) and the zero-age main sequence progenitor masses were mapped to compact remnants by $0.8<M / M_{\odot}<1.5 \rightarrow 0.6 M_{\odot}$ $\mathrm{WD}, 1.5<M / M_{\odot}<2.5 \rightarrow 0.7 M_{\odot} \mathrm{WD}, 2.5<M / M_{\odot}<8.0 \rightarrow 1.1 M_{\odot} \mathrm{WD}$, $8.0<M / M_{\odot}<30.0 \rightarrow 1.4 M_{\odot}$ NS, $30.0<M_{\star} / M_{\odot} \rightarrow 10 M_{\odot}$ SBH Alexander 2005). $\Delta$ was estimated for a light/heavy mass boundary $M_{L / H}=5 M_{\odot}$ (the exact choice of $M_{L / H}$ does not affect the value of $\Delta$ for the evolved PMFs).

$\dot{N}_{H} \propto \dot{r} r^{2} n_{H}(r)=$ const, so that the number density of the heavy stars must scale as $n_{H} \propto r^{-\left(9 / 2-\alpha_{L}\right)} \propto r^{-\alpha_{H}}$. It then follows that

$$
\alpha_{H}=9 / 2-\alpha_{L}=11 / 4 \quad\left(p_{H}=5 / 4\right) .
$$

Equivalently, this result can be obtained by expressing the Fokker-Planck equations explicitly in terms of the diffusion coefficients as function of energy (Lightman \& Shapiro 1977, Eqs. 51a,b), and solving them under the assumption that only the drift term (dynamical friction) contributes. In practice, it is unlikely that physical systems actually have $\Delta \ll 0.1(\$ 5)$; $\alpha_{H}=11 / 4$ should be considered an upper limit on the logarithmic slope of the number density distribution of the heavy stars.

\section{THE PRESENT DAY MASS FUNCTION}


The value of $\Delta$ (Eq. 10) depends on the population's PMF. So-called universal IMFs, which extend all the way from the brown dwarf boundary $M_{1} \sim 0.1 M_{\odot}$ to $M_{2} \gtrsim 100 M_{\odot}$ (e.g. the Salpeter 1955 IMF and its subsequent refinements, the Miller \& Scalo 1979 and Kroupa 2001 IMFs), result in evolved populations (old coeval star-bursts or continuously star forming populations) that naturally separate into two mass scales, the $\sim 1 M_{\odot}$ scale of low-mass main-sequence dwarfs, white dwarfs and neutron stars, and the $\sim 10 M_{\odot}$ scale of stellar black holes, and typically have $\Delta<0.1$ (Fig. 2). Such evolved populations are well-approximated by the simple 2mass population model.

Generally, 10 Gyr old, continuously star-forming populations with a power-law IMF, $\mathrm{d} N / \mathrm{d} M \propto M^{-\gamma}$, have $\Delta<1$ for $\gamma \gtrsim 1.8$, and $\Delta>1$ for $\gamma \lesssim 1.8$ (assuming $M_{L}=0.1 M_{\odot}$, $\left.M_{H}=120 M_{\odot}, M_{L / H}=5 M_{\odot}\right)$. This implies that even flatter IMFs than the Salpeter IMF $(\gamma=2.35)$ lead to strong mass segregation.

It is not clear what is the typical PMF, and the corresponding value of $\Delta$, in galactic nuclei. There are indications that star formation deep in the potential well of a MBH can be very different from that in the field (Levin \& Beloborodov 2003; Milosavljević \& Loeb 2004; Paumard et al. 2006; Levin 2007). For example, the volumeaveraged stellar population in the central few $\mathrm{pc}$ of the GC is reasonably well approximated by a 10 Gyr old, continuously star-forming population with a universal IMF (Alexander \& Sternberg 1999) and has $\Delta<0.1$. In contrast, a recent analysis of late type giants in the inner $\sim 1 \mathrm{pc}$ of the GC suggests that the IMF there could be a flat $\gamma \sim 0.85$ power-law (Maness et al. 2007). This corresponds to $\Delta>10$ in the inner $\sim 1 \mathrm{pc}$, if that region evolved as an isolated system. However, that is unlikely, since the PMF reflects not only the local star formation, but also the volume-averaged population in the inner few pc, which contributes unbound stars and stars that diffuse in phase space from lower to higher energies. It is therefore quite possible that in some galactic nuclei the weak mass segregation solution is realized, while in others the strong mass segregation, depending on the system-to-system scatter in the PMF.

\section{RESULTS}

Figure (3) shows an example of a steady state solution of the DFs calculated for a 2-mass system in the weak segregation regime with $M_{L} / M_{H}=10$ and $N_{H} / N_{L}=1(\Delta \simeq 13)$, neglecting the loss-cone term. While the logarithmic slopes of the DFs are not constant, they vary only slightly away from the boundaries at $x=0$ and $x_{D}$.

When comparing the DF with observations of stars in the GC, a relevant energy scale is $x \sim 10$, which corresponds to orbits with a semi-major axis of $a=r_{\star} / 2 x=0.1 \mathrm{pc}$ for $r_{\star}=2$ $\mathrm{pc}$ in the GC. On that scale the cusp is Keplerian, but there are still enough observed stars for meaningful statistics (e.g. Schödel et al. 2007). Figure (4 L) shows the local logarithmic slopes of the DFs, $p_{L}$ and $p_{H}$, at $x=10$, as function of $\Delta$, for the same mass ratios modeled by BW77, $M_{H} / M_{L}=1.5$, 3 and 10. The transition between the weak and strong mass segregation solutions at $\Delta \sim 1$ is clearly seen. It is interesting to note that the set of models calculated by BW77 all happen to lie at $\Delta \gtrsim 1$, which explains why they found that "...there are no dramatic changes in the shapes of the curves, despite the fact that the unbound population ratio was varied by a factor of 64.", and wrongly concluded that the weak segregation solution is a universal solution.

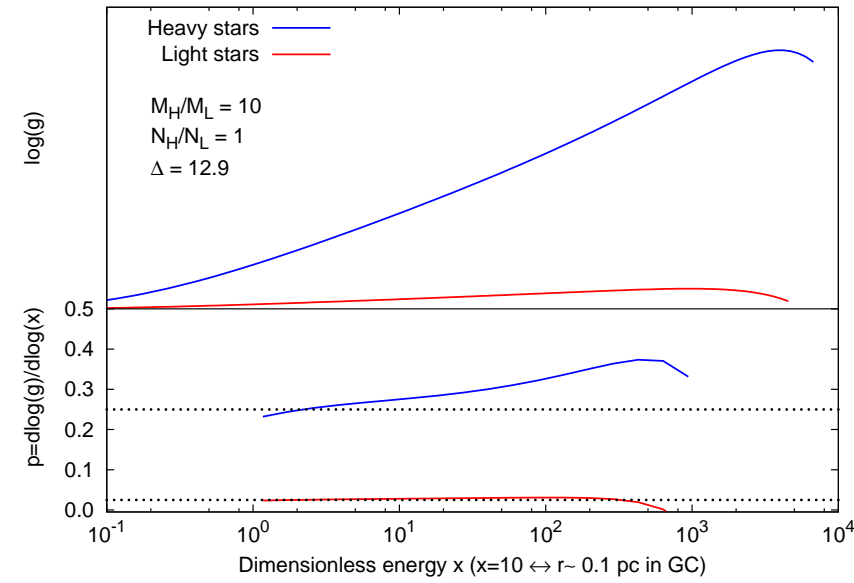

FIG. 3.- The steady state DF (on an arbitrary logarithmic scale) and its local logarithmic slope away from the boundaries, for $M_{H} / M_{L}=10$ and $N_{H} / N_{L}=1(\Delta=12.9)$. The loss-cone term is neglected. The boundary conditions are a sink at $x_{D}=10^{4}$ and violently relaxed (equal velocity dispersion) unbound stars. The asymptotic BW77 solution is indicated by the dotted lines $\left(p_{L}=1 / 40, p_{H}=7 / 4\right)$.

A useful average of the logarithmic slope is obtained from the corresponding stellar density curve (Eq. 9, cf Fig. 1), which can be directly related to the observable stellar density distribution. A comparison of Figs. (4 L) and (4 R) shows that the shape of the curves is hardly affected by the method chosen for deriving the logarithmic slopes. We also repeated these calculations with a loss-cone term (Eq. 4), and confirmed the BW77 conclusion that the effect on the shape of the DF curve is small, thereby justifying its omission.

The asymptotic limits $(\$ 3.1)$ are also clearly seen in Fig. (4). When $\Delta \rightarrow \infty$, the weak segregation BW77 solution holds, with $p_{H}=0.25$ and $p_{L}=M_{L} / 4 M_{H}$. When $\Delta \rightarrow 0$, the light stars, which dominate the population, assume the single mass population DF, $p_{L}=1 / 4$, while the heavy stars concentrate to the center. For low mass ratios, $M_{H} / M_{L} \lesssim 4$, where dynamical friction is less efficient, the heavy stars approximately obey the BW77 relation, $p_{H}=\left(M_{H} / M_{L}\right) p_{L}=M_{H} / 4 M_{L}$. For higher mass ratios, $M_{H} / M_{L} \gtrsim 4$, the heavy stars approach the dynamical friction limit, $p_{H} \rightarrow 5 / 4$.

The transition between the weak and strong mass segregation solutions is a reflection of the breakdown of the zero-flow assumption as $\Delta \rightarrow 0$. The dimensional scale $I_{\star}$ of the dimensionless stellar current into the $\mathrm{MBH}, Q_{M}(\varepsilon)=I_{M}(\varepsilon) / I_{\star}$, is $I_{\star} \sim O\left(N_{M_{\star}}\left(<r_{\star}\right) / t_{\star}\right)$ (note that when the loss-cone is neglected, the steady-state current is independent of energy). This is also the order of magnitude of the stellar current into the $\mathrm{MBH}$ in a single mass population out of equilibrium. However, Bahcall \& Wolf (1976, Eq. 63) show that, neglecting the loss-cone, the steady-state current in a single mass population is $Q_{M} \sim 8 / x_{D} \sim 0$, and that steady state zero-flows further imply that $p_{H}(\varepsilon) / M_{H}=p_{L}(\varepsilon) / M_{L}$ in multi-mass populations (Bahcall \& Wolf 1977, Eqs. 41-46). The relevant physical timescale for the heavy star current $Q_{H}$ is not the $M_{L}-$ $M_{L}$ interaction timescale $1 / t_{\star}=\Gamma_{L} \propto D_{L L s}+D_{L L f} \sim 4 N_{L} M_{L}^{2}$ (here we choose $M_{\star}=M_{L}$ ), but rather the rate of $M_{H}-M_{H}$ and $M_{L}-M_{H}$ interactions, $\Gamma_{H} \propto D_{H H s}+D_{L H s}+D_{H H f}+D_{L H f} \sim$ $N_{L} M_{L}^{2}\left[4+M_{H} / M_{L}+4 N_{H} M_{H}^{2} / N_{L} M_{L}^{2}\right]$. To compare meaningfully the heavy star current $Q_{H}$ across the range of our models, we rescale it to $1 / \Gamma_{H}$ instead of $t_{\star}$, and normalize by the 

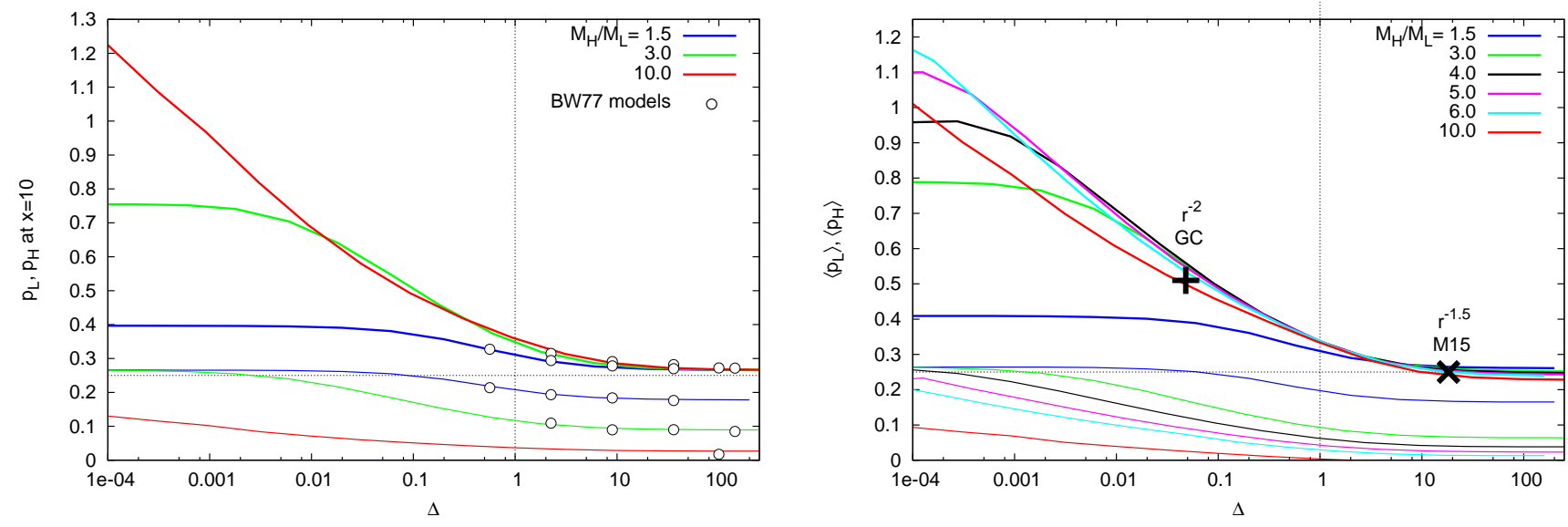

FIG. 4.- The power-law indices $p_{L, H}$ as functions of $\Delta$ for various mass ratios, assuming violent relaxation boundary conditions, and neglecting the loss-cone term. Left: The indices, as derived from the logarithmic slopes of $g_{L, H}$ at $x=10$, compared to the logarithmic slopes of the DFs calculated by Bahcall \& Wolf (1977). Right: The indices, as derived from the average logarithmic slopes of the densities $3 / 2+p_{L, H}$ in the range $r=0.003-0.3 \mathrm{pc}$ (for $r_{\star}=2 \mathrm{pc}$ ) with the expected $p_{H}$ for a model of the GC assuming continuous star formation with the Miller-Scalo IMF (Fig. 2 Alexander \& Sternberg 1999; Alexander 2005), and for a mass-function model of globular cluster M15 (Murphy et al. 1997), assuming it harbors an IMBH.

number of heavy stars to obtain the current per star,

$$
\widehat{Q}_{H}=Q_{H} \frac{N_{L}}{N_{H}} \frac{\Gamma_{L}}{\Gamma_{H}}=Q_{H} 4 \frac{N_{L}}{N_{H}} /\left(4+\frac{M_{H}}{M_{L}}+4 \frac{N_{H} M_{H}^{2}}{N_{L} M_{L}^{2}}\right) .
$$

Figure (5) shows $\widehat{Q}_{H}$ as function of $\Delta$ for different mass ratios. For $\Delta>1$, which is the range explored by BW77, we confirm their zero-flow result. In contrast, for $\Delta \ll 1$, we find that the zero-flow assumption no longer holds, $\widehat{Q}_{H} \gtrsim O(1)$, and the heavy stars sink to the $\mathrm{MBH}$ at the maximal possible rate, the dynamical friction rate. Figure (5) also shows that the physically required inclusion of a loss-cone term in the Fokker-Planck equations (Eq. 6) increases the current, but the energy averaged flow in the weak segregation limit $\Delta \gg 1$, while not quite as small as without a loss-cone, is still strongly suppressed $^{5}$. It then follows that the relation $p_{H} / M_{H} \sim p_{L} / M_{L}$ is approximately satisfied in this limit even with a loss-cone, as found by BW77 and confirmed here. In the limit strong segregation limit $\Delta \ll 1$, the dynamical friction-driven current of the heavy stars is insensitive to the presence or absence of a loss-cone, since $T_{\mathrm{df}} \sim\left(M_{L} / M_{H}\right) T_{R}$.

Figure (4R) shows the expected segregation of the massive stellar objects in the GC (based on the old, continuous starformation PMF model, Fig. 2), and in globular cluster M15, assuming it harbors an IMBH (based on the tentative PMF model of Murphy et al. 1997, which assumes that the most massive remnants in the cluster are $1.4 M_{\odot}$ neutron stars). The $\mathrm{GC}$ is expected to lie in the strong relaxation regime $(\Delta=$ $0.05)$, while M15 in the weak relaxation regime $(\Delta \sim 18)$.

We refrain here from a more detailed quantitative analysis of the numerical results because the exact values of $p_{L, H}(\Delta)$ depend somewhat on the way these are evaluated (locally, or from the density curve), and because the convergence of our numerical Fokker-Planck solver becomes progressively worse in the limits $\Delta \rightarrow 0$ and $M_{H} / M_{L} \gg 1$. However, any numerical inaccuracies in those limits are unlikely to have practical

5 In the presence of a loss-cone, the current in the weak segregation limit is set by the diffusion rate into the loss-cone at $x_{\text {diff }}(\$ 2), I_{H} \sim$ $N_{H}\left(x_{\text {diff }}\right) /\left[t_{H} \log \left(\left[J_{c}\left(x_{\text {diff }}\right) / J_{l c}\right)\right]\right.$, where $t_{H} \sim \Gamma_{H}^{-1}$ is the $\left(M_{H}\right.$-dominated $)$ relaxation time (cf Eq. 6]. It then follows that $\widehat{Q}_{H}\left(x_{\text {diff }}\right) \sim I_{H}\left(x_{\text {diff }}\right) /\left(N_{H} \Gamma_{H}\right) \sim$ $\left[1 /\left(2 x_{\text {diff }}\right)\right]^{3-\alpha_{H}} / \log \left[\left(c / \sigma_{\star}\right) / \sqrt{32 x_{\text {diff }}} \sim 0.01\right.$ for $x_{\text {diff }}=10$.

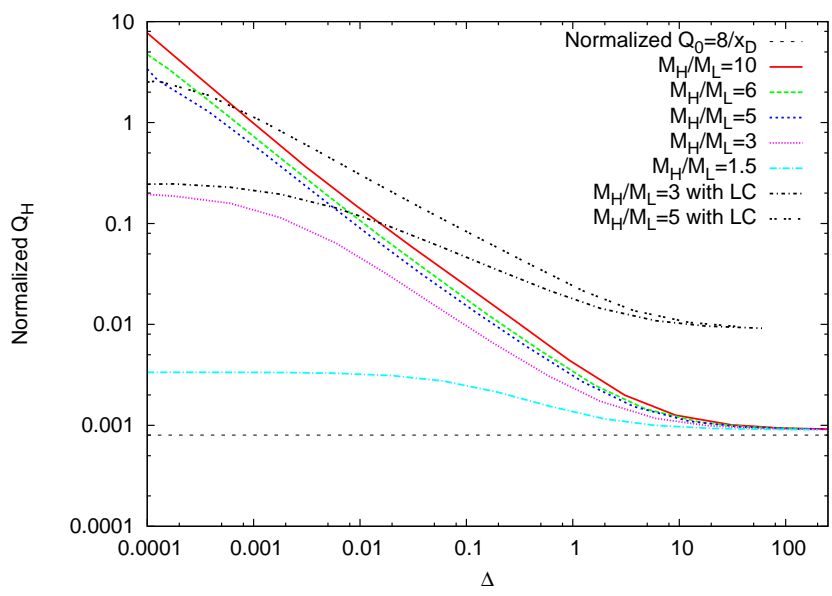

FIG. 5.- The normalized current of the heavy stars, $\widehat{Q}_{H}$ (Eq. 12), as function of the relaxational coupling parameter, $\Delta$. The horizontal line marks $\widehat{Q}_{0}=8 / x_{D}$, the zero-flow in a single mass population (Bahcall \& Wolf 1976). For $\Delta \gg 1, \widehat{Q}_{H} \sim \widehat{Q}_{0} \sim 0$. However, for $\Delta \ll 1$ in the strong mass-segregation regime, $\widehat{Q}_{H} \gtrsim O(1)$, indicating that massive stars flow inwards at the maximal possible rate, the dynamical friction rate. The transition between the two flow regimes is also apparent in the energy-averaged currents in models with a loss-cone term.

implications, since realistic stellar systems are not expected to have $\Delta \lesssim 0.01$ (a possible exception could be the hypothesized steady flow of IMBHs from dense stellar clusters to the $\mathrm{MBH}$, Portegies Zwart et al. 2006).

\section{DISCUSSION AND SUMMARY}

\subsection{Strong segregation and other relaxation processes}

Strong mass segregation, or mass segregation instability, shares some common features with the Spitzer, or equipartition, instability in a cluster (Spitzer 1969), where the heavy stars decouple from the light ones and evolve away from equipartition by forming a dense sub-system in the center with a much higher velocity dispersion, $\sigma_{H}^{2} / \sigma_{L}^{2}>M_{L} / M_{H}$. However, these two instabilities are distinct effects. The Spitzer instability occurs only when the heavy stars are relatively common in the population, $N_{H} M_{H}^{5 / 2} / N_{L} M_{L}^{5 / 2}>\beta$, where $\beta \simeq 0.16$ (for $M_{H} \gg M_{L}$ and $N_{H} M_{H} \ll N_{L} M_{L}$ ). In contrast, in the Keple- 
rian potential near a $\mathrm{MBH}$, the Jeans equation dictates that $\sigma_{H}^{2} / \sigma_{L}^{2}=\left(5 / 2+p_{L}\right) /\left(5 / 2+p_{H}\right) \sim 1$ (Alexander \& Kumar 2001), and so equipartition is never achieved, irrespective of the heavy-to-light mass or number ratios. Strong mass segregation is an instability in the spatial distribution of the heavy stars, which occurs in the opposite limit to the Spitzer instability, when the heavy stars are relatively rare in the population, $\Delta \sim N_{H} M_{H} / N_{L} M_{H}<1$ (for $M_{H} \gg M_{L}$, Eq. 10).

Perets et al. (2007) focused on the relaxation of light objects, stars, by heavy objects, massive perturbers (e.g. giant molecular clouds or clusters, with $M_{H} \gg M_{L}$ ), and expressed the efficiency of massive perturber-induced relaxation relative to star-star relaxation by the parameter $\mu_{2}=N_{H} M_{H}^{2} / N_{L} M_{L}^{2}$ $\left(\sim\left(D_{H L s}+D_{H L f}\right) /\left(D_{L L s}+D_{L L f}\right) \simeq 3 N_{H} M_{H}^{2} / 4 N_{L} M_{L}^{2}\right)$. When $\mu_{2} \gg 1$, massive perturbers dominate stellar relaxation. Here we focus on the dynamics of the heavy objects, the SBHs, and so the relaxational coupling parameter $\Delta$ is defined to also take into account the interactions between the heavy masses. The parameter $\mu_{2}$ addresses the question "Which mass component dominates the relaxation of the light stars?", while $\Delta$ addresses the question "Which mass component dominates the dynamics of the heavy stars?". The two are related by $\Delta=\mu_{2} 4 /\left(3+M_{H} / M_{L}\right)$.

Our approximate treatment of the mass segregation process neglects the relaxation of angular momentum to near radial ("loss-cone") orbits. This, rather than diffusion in energy, is the primary channel for stellar destruction by the MBH. A full treatment of the mass segregation problem in $(E, J)$ phase space (e.g. Cohn \& Kulsrud 1978) is beyond the scope of this work. Here we follow BW77, who treated the problem approximately in $E$ only, and who further showed that the neglect of an effective loss-cone term (Eq. 4) did not much change the shape of the DFs. We confirmed that this conclusion also holds for our mass segregation models (Fig. 5).

\subsection{Possible implications of strong mass segregation}

Strong mass segregation occurs in stellar systems with a relatively lower fraction of SBHs, that reach a higher central concentration of SBHs very close to the $\mathrm{MBH}$, compared to systems with a higher fraction of SBHs that undergo weak segregation. To compare two such systems, and to determine which has more SBHs enclosed inside some given volume around the $\mathrm{MBH}$, it is necessary to specify the comparison procedure (e.g. assuming an equal total stellar mass; or the same $\mathrm{MBH}$ mass and the $M_{\bullet} / \sigma$ relation; or an equal number of SBHs within $r_{h}$ ). The choice depends on the question of interest. Here we do not address such quantitative issues, but limit ourselves to briefly listing some processes that are expected to be affected by the degree of segregation.

Accelerated relaxation. The degree of mass segregation affects both the non-coherent 2-body relaxation timescale, which scales as $1 / \int M^{2}(\mathrm{~d} N / \mathrm{d} M) \mathrm{d} M$ (see $\$ 1$, and the resonant relaxation timescale, which scales as as $\int M(\mathrm{~d} N / \mathrm{d} M) \mathrm{d} M / \int M^{2}(\mathrm{~d} N / \mathrm{d} M) \mathrm{d} M$ Rauch \& Tremaine 1996). In particular, the stronger the mass segregation, the shorter is the resonant relaxation timescale, which does not depend on the number of stars, but only on their typical mass. Efficient resonant relaxation near the $\mathrm{MBH}$ may affect stellar orbits and accretion disk dynamics there (see below).

$G W$ event rates. The GW EMRI rate is determined by the number of potential GW sources within the critical radius $r_{\text {crit }}$, which demarcates the boundary between compact object that inspiral into the $\mathrm{MBH}$ those that plunge (infall) into it. The critical radius is a function of the relaxation time, and to good approximation the EMRI rate is $\Gamma \sim N\left[r_{\text {crit }}\left(T_{R}\right)\right] / T_{R}$ (Hopman \& Alexander 2005). Strong segregation will affect the EMRI rate both by modifying the 2-body relaxation time and by affecting the number of stars enclosed inside $r_{\text {crit }}$, as well as by decreasing the resonant relaxation timescale. Similarly, the rates of detectable GW bursts from fly-bys near the Galactic MBH strongly depend on the number of SBHs near it (Hopman et al.2007). Strong segregation may also affect GW emission from close $\mathrm{SBH}-\mathrm{SBH}$ interactions in a very dense cusp (O’Leary et al. 2008).

SBH-star interactions. A higher central concentration of SBHs affects the probability of SBH-star interactions, which can lead to the randomization of stellar orbits, the heating of a stellar disk (Perets et al. 2008), the 3-body exchange capture of massive young stars near the $\mathrm{MBH}$ (Alexander \& Livio 2004), or the ejection of hyper-velocity stars (O'Leary \& Loeb 2008).

SBH-accretion disk interactions. A higher central concentration of SBHs within $10^{1-3}$ gravitational radii of the $\mathrm{MBH}$ could exert coherent torques on the accretion disk, warp it and possibly affect its hydrodynamics (Bregman \& Alexander, 2008 , in prep.). The SBHs may shock the disk as they cross it, experience drag by it, and be carried by it to the MBH (e.g. Artymowicz et al. 1993; Nayakshin et al. 2004; Subr \& Karas 2005).

Enhanced gravitational lensing. SBHs projected near the Einstein angle of the MBH can strongly modify the gravitational lensing properties of the $\mathrm{MBH}$, in a way similar to the effect of a planet orbiting a Galactic star that is lensing a background source (Alexander \& Loeb 2001; Chanamé et al. 2001).

\subsection{Summary}

We show that the steady state solution of a relaxed multimass stellar system around a $\mathrm{MBH}$ has two branches: the known weak (Bahcall-Wolf) mass segregation solution, where the difference in the degree of central concentration of the light and heavy stars is relatively small, and a newly discovered strong segregation solution, where the difference is much larger. The nature of the solution is determined by the global properties of the system (the mass ratio between the heavy and light stars, $M_{H} / M_{L}$, and their number ratio far from the $\mathrm{MBH}, N_{H} / N_{L}$ ) through the relaxational coupling parameter, $\Delta=4 N_{H} M_{H}^{2} /\left[N_{L} M_{L}^{2}\left(3+M_{H} / M_{L}\right)\right]$. Strong mass segregation occurs when the heavy stars are relatively rare in the population $(\Delta \ll 1)$, and sink to the center by dynamical friction. Weak mass segregation occurs when the heavy stars are common in the population $(\Delta \gg 1)$ and settle to the single mass stellar cusp solution. We show that relaxed old coeval or continuously star-forming populations with a universal IMF typically have $\Delta<0.1$, and thus settle to the strong mass segregation solution around a $\mathrm{MBH}$.

We are grateful to S. Tremaine and M. Freitag for useful discussions. TA is supported by ISF grant 928/06, ERC Starting Grant 202996 and a New Faculty grant by Sir H. Djangoly, CBE, of London, UK. CH is supported by a Veni scholarship from the Netherlands Organization for Scientific Research (NWO). 


\section{REFERENCES}

Alexander, T. 1999, ApJ, 527, 835

-. 2005, Phys. Rep., 419, 65

Alexander, T. 2007, in 2007 STScI Spring Symposium: Black Holes, ed.

M. Livio \& A. Koekemoer (Cambridge University Press), in press, arXiv:astro-ph/0708.0688

Alexander, T. \& Kumar, P. 2001, ApJ, 549, 948

Alexander, T. \& Livio, M. 2004, ApJ, 606, L21

Alexander, T. \& Loeb, A. 2001, ApJ, 551, 223

Alexander, T. \& Sternberg, A. 1999, ApJ, 520, 137

Amaro-Seoane, P., Gair, J. R., Freitag, M., Miller, M. C., Mandel, I., Cutler,

C. J., \& Babak, S. 2007, Classical and Quantum Gravity, 24, 113

Artymowicz, P., Lin, D. N. C., \& Wampler, E. J. 1993, ApJ, 409, 592

Bahcall, J. N. \& Wolf, R. A. 1976, ApJ, 209, 214

-. 1977, ApJ, 216, 883

Baumgardt, H., Makino, J., \& Ebisuzaki, T. 2004, ApJ, 613, 1143

Binney, J. \& Tremaine, S. 1987, Galactic Dynamics (Princeton, NJ: Princeton University Press)

Boroson, T. A., Thompson, I. B., \& Shectman, S. A. 1983, AJ, 88, 1707

Chanamé, J., Gould, A., \& Miralda-Escudé, J. 2001, ApJ, 563, 793

Chandrasekhar, S. 1943, ApJ, 97, 255

Cohen, J. G. 1986, AJ, 92, 1039

Cohn, H. \& Kulsrud, R. M. 1978, ApJ, 226, 1087

de Grijs, R. \& Peletier, R. F. 2000, MNRAS, 313, 800

Deegan, P. \& Nayakshin, S. 2007, MNRAS, 377, 897

Eisenhauer, F. et al. 2005, ApJ, 628, 246

Ferrarese, L. \& Merritt, D. 2000, ApJ, 539, L9

Freitag, M., Amaro-Seoane, P., \& Kalogera, V. 2006a, ApJ, 649, 91

Freitag, M., Gürkan, M. A., \& Rasio, F. A. 2006b, MNRAS, 368, 141

Freitag, M., Rasio, F. A., \& Baumgardt, H. 2006c, MNRAS, 368, 121

Gebhardt, K. et al. 2000, ApJ, 539, L13

Genzel, R., Pichon, C., Eckart, A., Gerhard, O. E., \& Ott, T. 2000, MNRAS, 317,348

Genzel, R. et al. 2003, ApJ, 594, 812

Ghez, A. M., Salim, S., Hornstein, S. D., Tanner, A., Lu, J. R., Morris, M., Becklin, E. E., \& Duchêne, G. 2005, ApJ, 620, 744

Ghez, A. M. et al. 2008, ApJ, accepted (astro-ph/0808.2870)

Giacconi, R., Murray, S., Gursky, H., Kellogg, E., Schreier, E., \& Tananbaum, H. 1972, ApJ, 178, 281

Gillessen, S., Eisenhauer, F., Trippe, S., Alexander, T., Genzel, R., Martins, F., \& Ott, T. 2008, ApJ, submitted

Hopman, C. 2006, in AIP Conf. Proc. 873: laser interferometer space antenna: Sixth International LISA Symposium, ed. Merkowitz, S. M. and Livas, J. C. (AIP), (astro-ph/0608460)

Hopman, C. \& Alexander, T. 2005, ApJ, 629, 362

-. 2006a, ApJ, 645, 1152

-. 2006b, ApJ, 645, L133

Hopman, C., Freitag, M., \& Larson, S. L. 2007, MNRAS, 378, 129

Kroupa, P. 2001, MNRAS, 322, 231

Kroupa, P., Tout, C. A., \& Gilmore, G. 1993, MNRAS, 262, 545

Levi, M. 2006, Master's thesis, Weizmann Institute of Science

Levin, Y. 2007, MNRAS, 374, 515

Levin, Y. \& Beloborodov, A. M. 2003, ApJ, 590, L33

Lightman, A. P. \& Shapiro, S. L. 1977, ApJ, 211, 244
Lynden-Bell, D. 1967, MNRAS, 136, 101

-. 1969, Nature, 223, 690

Maness, H. et al. 2007, ApJ, 669, 1024

Matthews, T. A. \& Sandage, A. R. 1963, ApJ, 138, 30

Merritt, D., Mikkola, S., \& Szell, A. 2007, submitted to ApJ (arXiv:astro-ph/0705.2745)

Miller, G. E. \& Scalo, J. M. 1979, ApJS, 41, 513

Miller, M. C. \& Hamilton, D. P. 2002, MNRAS, 330, 232

Milosavljević, M. \& Loeb, A. 2004, ApJ, 604, L45

Miralda-Escudé, J. \& Gould, A. 2000, ApJ, 545, 847

Morris, M. 1993, ApJ, 408, 496

Mouawad, N., Eckart, A., Pfalzner, S., Schödel, R., Moultaka, J., \& Spurzem, R. 2005, Astronomische Nachrichten, 326, 83

Muno, M. P., Pfahl, E., Baganoff, F. K., Brandt, W. N., Ghez, A., Lu, J., \& Morris, M. R. 2005, ApJ, 622, L113

Murphy, B. W., Cohn, H. N., Lugger, P. M., \& Drukier, G. A. 1997, in Bulletin of the American Astronomical Society, Vol. 29, Bulletin of the American Astronomical Society, 1338-+

Nayakshin, S., Cuadra, J., \& Sunyaev, R. 2004, A\&A, 413, 173

O'Leary, R. M., Kocsis, B., \& Loeb, A. 2008, MNRAS, submitted

O'Leary, R. M. \& Loeb, A. 2008, MNRAS, 383, 86

Paumard, T., Genzel, R., Martins, F., Nayakshin, S., Beloborodov, A. M., Levin, Y., Trippe, S., Eisenhauer, F., Ott, T., Gillessen, S., Abuter, R., Cuadra, J., Alexander, T., \& Sternberg, A. 2006, Journal of Physics Conference Series, 54, 199

Peebles, P. J. E. 1972, ApJ, 178, 371

Perets, H. B., Gualandris, A., Merritt, D., \& Alexander, T. 2008, in The Central Kiloparsec: Active Galactic Nuclei and Their Hosts (Memorie della Societa Astronomica Italiana), in press

Perets, H. B., Hopman, C., \& Alexander, T. 2007, ApJ, 656, 709

Portegies Zwart, S. F., Baumgardt, H., McMillan, S. L. W., Makino, J., Hut, P., \& Ebisuzaki, T. 2006, ApJ, 641, 319

Quinlan, G. D. 1996, New Astronomy, 1, 255

Rauch, K. P. \& Tremaine, S. 1996, New Astronomy, 1, 149

Rees, M. J. 1984, ARA\&A, 22, 471

-. 1988, Nature, 333, 523

Salpeter, E. E. 1955, ApJ, 121, 161

Schaller, G., Schaerer, D., Meynet, G., \& Maeder, A. 1992, A\&AS, 96, 269

Schmidt, M. 1963, Nature, 197, 1040

Schödel, R., Ott, T., Genzel, R., Eckart, A., Mouawad, N., \& Alexander, T. E. 2003, ApJ, 596, 1015

Schödel, R. et al. 2007, A\&A, 469, 125

Shapiro, S. L. \& Marchant, A. B. 1978, ApJ, 225, 603

Spitzer, L. 1987, Dynamical evolution of globular clusters (Princeton, NJ,

Princeton University Press, 1987)

Spitzer, L. J. 1969, ApJ, 158, L139+

Syer, D. \& Ulmer, A. 1999, MNRAS, 306, 35

Šubr, L. \& Karas, V. 2005, A\&A, 433, 405

Wolfe, A. M. \& Burbidge, G. R. 1970, ApJ, 161, 419

Wyller, A. A. 1970, ApJ, 160, 443

Young, P. 1980, ApJ, 242, 1232 